\title{
Federated Learning Assisted Multi-UAV Networks
}

\author{
Hongming Zhang, Member, IEEE, and Lajos Hanzo, Fellow, IEEE
}

\begin{abstract}
Unmanned aerial vehicles (UAVs) have been recognized as a promising technology to be used in a wide range of civilian, public and military applications. However, given their limited payload and flight time, multiple UAVs may have to be harnessed for accomplishing complex high-level tasks, where a control center can be employed for coordinating their actions. In this paper, we consider image classification tasks in UAV-aided exploration scenarios, where the coordination of multiple UAVs is implemented by a ground fusion center (GFC) positioned in a strategic, but inaccessible location, such as a mountain top, where recharging the battery is uneconomical or may even be infeasible. On-board cameras are carried by each UAV and then, federated learning (FL) is invoked for reducing the communication cost between the UAVs and the GFC, and the computational complexity imposed on the GFC. In our proposed FL-aided classification approach, initially local training is performed by each UAV based on the locally collected images to create a local model. Then, each UAV sends its locally acquired model to the GFC via a fading wireless channel, where a global model is generated, which is then fed back to each UAV for the next round of their local training. In order to further minimize the computational complexity imposed on the GFC by the UAVs, weighted zero-forcing (WZF) transmit precoding (TPC) is used at each UAV based on realistic imperfect channel state information (CSI). The system performance attained is evaluated by simulations, showing that the proposed system is capable of attaining a high classification accuracy at relatively low communication cost.
\end{abstract}

Index Terms-Federated learning, unmanned aerial vehicle, multi-class classification, convolutional neural network, deep learning, imperfect CSI,

\section{INTRODUCTION}

Due to their ease of deployment and high agility, unmanned aerial vehicles (UAVs), also commonly known as drones or remotely piloted aircraft, have been considered as enablers of various applications [1-3]. Typically, UAVs can be classified into two categories, namely fixed-wing and rotary-wing UAVs [4]. Fixed-wing UAVs are capable of high-speed flight despite carrying heavy payloads in transportation applications. By contrast, rotary-wing UAVs with limited velocity and payload are more suitable for stationary communications applications in the absence of base station (BS) coverage [5]. As a benefit

This work was supported in part by the Fundamental Research Funds for the Central Universities under Grant 500420837 and Grant 505020134. Corresponding author: Hongming Zhang

L. Hanzo would like to acknowledge the financial support of the Engineering and Physical Sciences Research Council projects EP/N004558/1, EP/P034284/1, EP/P034284/1, EP/P003990/1 (COALESCE), of the Royal Society's Global Challenges Research Fund Grant as well as of the European Research Council's Advanced Fellow Grant QuantCom.

H. Zhang is with the School of Software Engineering, Beijing University of Posts and Telecommunications, Beijing 100876, China (e-mail: zhanghm@bupt.edu.cn)

L. Hanzo is with the School of Electronics and Computer Science, University of Southampton, Southampton, SO17 1BJ, UK (e-mail: lh@ecs.soton.ac.uk) of these features, UAVs have been employed for supporting wireless connectivity, hence expanding the network coverage.

In many applications, multiple UAVs are required for accomplishing complex high-level tasks, such as search and rescue operations in disaster scenarios, target monitoring, information dissemination, an so on [6-11]. In a multiUAV system, the UAVs operate in a coordinated manner to support reliable and efficient communications. Typically, the coordination of UAVs can be performed with the aid of a ground control station (GCS). For example, a multi-UAV system can be controlled by a ground base station (BS) in an urban area having good mobile network coverage. By contrast, in a rural area having poor network coverage, multi-UAV systems can be coordinated by a control station dropped off by a UAV on an inaccessible mountain-top or in a desert for acting as a ground fusion center (GFC), where recharging the battery may be impractical. By considering the limited computing and communication capabilities of the GFC, it is challenging to design an efficient GFC-aided multi-UAV system, especially when performing complex tasks that impose high computational and communication costs. For example, in an aerial exploration scenario, computer vision tasks have to be carried out by a GFC-aided multi-UAV system, where thousands of high-quality images and videos are collected by UAVs and are then sent to the GFC for analysis. In this case, the limited-capability GFC may be overwhelmed by the processing requirements.

Recently, federated learning (FL), which is an emerging decentralized machine learning technique $[12,13]$, has been conceived for handling similar problems, where a model is learned by each participating device (client) based on its local dataset. Then, the local model updates are sent to a parameter server for updating a shared global model. Finally, the updated global model is fed back to UAVs for the next round of local training. In contrast to the conventional scenario of sending back the training datasets to the server, local model updates are sent back to the server in FL. Hence, training models are shared and the privacy is preserved, whilst the communication cost can be reduced. Given these compelling benefits, increasing research attention has been dedicated to applying $\mathrm{FL}$ in wireless communications [14-18]. In [14], FL was invoked in resource-constrained mobile edge computing systems, while in [15], it was applied to Internet of Things (IoT) scenarios. Kang et al. [16] proposed a reputation-based worker selection scheme for improving the learning performance of FL, where sophisticated blockchain aided techniques were conceived for secure reputation management. In [17], FL was harnessed for scheduling and inter-cell interference management problems encountered in wireless networks, showing that an improved performance can be attained, provided that the signal-tointerference-plus-noise ratio (SINR) is sufficiently high. Sama- 
rakoon et al. [18] proposed a distributed FL technique for solving a joint power and resource allocation problem in the ultra-reliable low-latency communication (URLLC) scenario of vehicular networks, demonstrating that the power consumption of vehicular users can be beneficially reduced.

Against the above background, the new contributions of this paper are summarized as follows:

- We propose FL-aided multi-UAV systems for performing classification tasks in the broad application area of exploration scenarios. In our proposed system, multiple UAVs are coordinated by a GFC to form a cooperative multi-UAV network. Each UAV is assumed to be equipped both with computing-caching-communication devices and with a camera, where the geographic area is surveyed by the on-board cameras.

- The distributed FL philosophy is appropriately adapted for accomplishing the image classification tasks, where initially local training is carried out by each UAV relying on its own locally collected images. Then, the model gleaned from the local training is transmitted to the GFC over fading wireless channels. Finally, a global model is constructed at the GFC based on all the received local models, which is then fed back to each UAV for the next local model update procedure.

- In order to facilitate global model updates at the GFC, weighted zero-forcing (WZF) transmit precoding (TPC) is used at each UAV for mitigating the interference imposed on the GFC, where the effect of realistic imperfect CSI is considered. Finally, the overall system performance is evaluated, demonstrating that the proposed system is capable of attaining a high image classification accuracy at relatively low communication cost.

The rest of the paper is organized as follows. In Section II, we describe the system model. The proposed FL-aided image classification scheme is detailed in Section III, while our simulation results are discussed in Section IV. Finally, we offer our conclusions in Section V.

\section{SYSTEM MODEL}

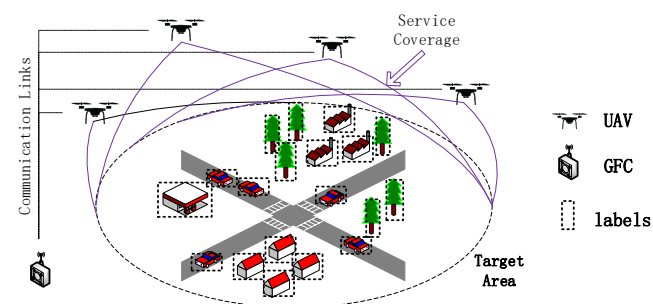

Fig. 1: Illustration of a multi-UAV system designed for performing image classification tasks in a geographic area exploration scenario, where the coordination of UAVs relies on a GFC.

As illustrated in Fig. 1, we consider image classification tasks in a geographic area exploration scenario, where $U$ UAVs hovering at a certain altitude are supported by a GFC. Again, the GFC is in a hard-to-reach but strategically important position, for example at a mountain-top. The geographic area to be explored is denoted as $\mathcal{A}$, which is uniformly divided into $Q$ sub-areas denoted as $\mathcal{A}=\left\{\mathcal{A}_{0}, \ldots, \mathcal{A}_{Q-1}\right\}$. Each sub-area is first surveyed by UAVs equipped with on-board cameras, for example for spotting wild-life or trees to be sprayed by pesticide. Note here that the range of each sub-area $\mathcal{A}_{q}$ is dependent on the image resolution requirements of the optical camera for each sub-area $q=0, \ldots, Q-1$. During each subarea's exploration, multiple UAVs are deployed at different locations in order to avoid any blind spot problems and for collecting more images during a certain mission execution time than a single UAV could. The whole area $\mathcal{A}$ can be searched by the UAVs relying on optimized trajectories given in $[10,19]$. Each UAV is assumed to be equipped with a single antenna, a caching device as well as a processor. Again, the UAVs communicate with the GFC via a fading wireless link. For the sake of simplicity, a star topology is adopted by our multi-UAV network, where all UAVs are connected directly to the resource-limited GFC. Here, we assume that the GFC is always within the communication range of UAVs. Naturally, the radio frequency communication range within $\mathcal{A}$ is much higher than the optical camera range within each sub-area $\mathcal{A}_{q}$ for $q=0, \ldots, Q-1$.

\section{A. Channel Model}

We assume that the complex-valued channel envelope between a UAV and the GFC is time-invariant during a transmission block, but fades independently from block to block. Furthermore, the channel between a UAV and the GFC can be modeled by the air-to-ground (A2G) channel model of [20,21]. Typically, the A2G channel operates in LOS/NLOS modes with probabilities of $p_{\mathrm{LoS}}=\{1+$ $\left.\rho_{1} \exp \left(-\rho_{2}\left[(180 / \pi) \arctan \left(H_{\mathrm{B}} / d\right)-\rho_{1}\right]\right)\right\}^{-1}$ and $p_{\mathrm{NLoS}}=$ $1-p_{\mathrm{LoS}}$, where $\rho_{1}$ and $\rho_{2}$ are constants that are characteristic of the environment (rural, urban, dense urban, or others), $d$ is the distance between the UAV and the GFC, while $H_{\mathrm{B}}$ is the building height in meters following the Rayleigh distribution of [20] $p\left(H_{\mathrm{B}}\right)=\left(H_{\mathrm{B}} / \gamma^{2}\right) \exp \left\{-H_{\mathrm{B}} /\left(2 \gamma^{2}\right\}\right.$, where $\gamma$ is an environment-dependent parameter.

Generally, the pathloss experienced in line-of-sight (LoS) and non-line-of-sight (NLoS) conditions can be considered separately. Explicitly, the channel's power gain between the $u$ th UAV and the GFC at time instant $t$ can be expressed as

$g_{u}(t)=\left(4 \pi f_{\mathrm{c}} / c\right)^{-2} d_{u}(t)^{-\eta}\left[p_{\mathrm{LoS}} \mu_{\mathrm{LoS}}+p_{\mathrm{NLoS}} \mu_{\mathrm{NLoS}}\right]^{-1}$,

where $f_{c}$ is the carrier frequency, $c$ is the speed of light, $d_{u}(t)$ is the distance between the $u$ th UAV and the GFC at time instant $t$, and $\eta$ is the pathloss exponent. In (1), $\mu_{\mathrm{LoS}}$ and $\mu_{\mathrm{NLOS}}$ are the attenuation factors of the LoS and NLoS links, respectively. According to [22, 23], the discretetime narrowband complex channel gain of the link between the $u$ th UAV and the GFC at time $t$ is given by

$$
h_{u}(t)=\sum_{n=0}^{N_{\mathrm{p}}-1} \sqrt{g_{u, n}(t)} e^{-j \phi_{u, n}(t)},
$$


where $N_{\mathrm{p}}$ is the total number of multipath components, $g_{u, n}(t)$ is the power gain of the $n$th multipath component given in (1), while $\phi_{n}(t)$ denotes the phase of the $n$th multipath component at time instant $t$.

\section{B. Communications Model}

In this paper, we focus our attention on what we refer to as the uplink transmission from the UAVs to the GFC, and we assume that the downlink transmission from the GFC to UAVs is implemented via an error-free shared link, whilst the uplink transmission from the UAVs to the GFC is achieved over a fading wireless channel. This is reasonable since in our system the GFC simply broadcast the same global model updates to the UAVs in the downlink, where a much simpler transmission technique is required, as it will be shown in Section III-B-b.

We consider a low-Doppler fading channel, where the transmit block-duration is relatively short in comparison to the channel's coherence time [24]. Moreover, perfect symbol-level synchronization is assumed among all the UAVs. As it will be detailed in Section III-B-b, the UAVs transmit simultaneously over the whole available bandwidth for the sake of simplicty. Let us denote the symbols transmitted by the $u$ th UAV as $x_{u}$, where we have $\mathbb{E}\left[\left|x_{u}\right|^{2}\right]=P_{\mathrm{Tx}}, \forall u$. Hence, the complex baseband symbols received at the GFC can be expressed as

$$
r=\sum_{u=1}^{U} h_{u} x_{u}+n,
$$

where $h_{u}$ is the sampled channel gain of the link between the $u$ th UAV and the GFC shown in Section II-A, while $n \sim$ $\mathcal{C N}\left(0, \sigma_{\mathrm{n}}^{2}\right)$ denotes the complex white Gaussian noise.

\section{Federated LeARning Aided Image CLASSIFICATION}

\section{A. Problem Statement}

As shown in Section II, we focus our attention on an image classification problem, where a total of $C$ classes have to be identified. Our goal is to learn a model defined by a weight space denoted as $\mathcal{W}$ from the training image-sets collected by the $U$ UAVs relying on the optimization problem of

$$
\min _{\mathcal{W}} \mathcal{L}\left(\mathcal{W} ; \mathcal{D}_{1}, \ldots, \mathcal{D}_{U}\right)
$$

where the local image-set is denoted as $\mathcal{D}_{u}=\left\{\left(\boldsymbol{a}_{u, m}, \boldsymbol{y}_{u, m}\right)\right.$ : $m=1, \ldots, M\}$ with $\left(\boldsymbol{a}_{u, m}, \boldsymbol{y}_{u, m}\right)$ being the $m$ th inputoutput data pair at the $u$ th UAV. Here, the training image-set $\boldsymbol{a}_{u, m}$ corresponds to the label vector $\boldsymbol{y}_{u, m} \in\{0,1\}^{C}$, where $y_{u, m}(c)=1$ indicates that the $m$ th observation is deemed to be from class $c$.

In theory, all the local observations collected by the UAVs could be sent to the GFC. Then, the GFC may collect all observations of all UAVs and create a global model with the aid of an appropriate machine learning algorithm. However, this might be impractical in a real-world setting due to the following reasons. Firstly, the number of observations to be transmitted by each UAV is excessive, leading to excessive UAV-GFC transmission requirements, which might be impractical due to the limited bandwidth and energy. Secondly, it is hard for the
GFC to cache all observations as well as to train a machine learning model having a sufficiently high image classification accuracy, due to its limited computing-caching-communication capability. In order to tackle the above-mentioned problem, FL is applied to reduce the communication cost between the UAVs and the GFC, as well as to alleviate both the caching and computational complexity imposed on the GFC. In our proposed FL-aided image classification approach, solely the model weights are sent to and processed at the GFC, rather than transferring the raw images.

\section{B. FL-aided Image Classification}

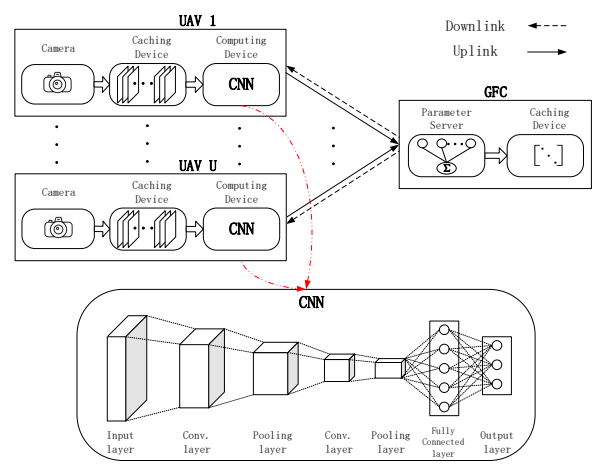

Fig. 2: Illustration of FL-aided image classification relying on our multiUAV system, where a convolutional neural network (CNN) is employed at each UAV for carrying out the local model update.

Federated learning can be invoked for addressing the abovementioned issue by training a shared global model from a federation of UAVs under the coordination of the GFC. Explicitly, it relies on a local model update procedure at each UAV and a weighted averaging procedure in the parameter server located at the GFC, as shown in Fig. 2. In particular, the local model update is performed at each UAV by a convolutional neural network (CNN), which has a width $D$, depth $J$, and input dimension $L$ in terms of the number of nodes. As seen in Fig. 2, the images stored in the caching device of each UAV, which are collected as a local training image-set, are input into the CNN for the sake of constructing a model based on the target labels at the output of the CNN. At the beginning, the global weights, which are initialized at the parameter server, are sent to the $U$ UAVs as the initial local weights. Then, the local weight matrices are updated at each UAV, as detailed below.

Since each UAV has its local training image-set (i.e., only a small fraction of the global training set), the minimization of (4) can be carried out by the iterative stochastic gradient descent (SGD) algorithm, where the gradient matrix of each UAV is updated at each iteration based on the global gradient parameter matrix obtained from the parameter server with respect to its own local image-set, as detailed below.

a) Local Update: Let us define the weight space of the $\mathrm{CNN}$ at the $u$ th UAV as $\mathcal{W}_{u}=\left\{\boldsymbol{W}_{u, m}^{0}, \boldsymbol{W}_{u, m}^{1}, \ldots, \boldsymbol{W}_{u, m}^{J-1}\right\}$, where we have $\boldsymbol{W}_{u, m}^{0} \in \mathbb{C}^{D \times L}, \boldsymbol{W}_{u, m}^{j} \in \mathbb{C}^{D \times D}$ for $j=$ $1, \ldots, J-2$, and $\boldsymbol{W}_{u, m}^{J-1} \in \mathbb{C}^{C \times D}$. During each local update of the CNN shown in Fig. 2, the output of the $j$ th layer can 
be expressed as $\boldsymbol{a}_{u, m}^{j}=\Xi\left(\boldsymbol{W}_{u, m}^{j-1} \boldsymbol{a}_{u, m}^{j-1}\right), j=1, \ldots, J$, where, we have $\boldsymbol{a}_{u, m}^{0}=\boldsymbol{a}_{u, m}$ and $\Xi(x)=1 /\left(1+e^{-x}\right)$ is the sigmoid function. Then, the loss function of the CNN at the $u$ th UAV can be expressed as

$$
\begin{aligned}
\mathcal{L}\left(\left\{\boldsymbol{W}_{u, m}^{j}\right\}_{j=0}^{J-1}\right)= & \frac{1}{M} \sum_{m=1}^{M} \sum_{c=1}^{C}-y_{u, m}(c) \log \widetilde{y}_{u, m}(c) \\
& -\left[1-y_{u, m}(c)\right] \log \left[1-\widetilde{y}_{u, m}(c)\right],
\end{aligned}
$$

where $\widetilde{y}_{u, m}(c)=a_{u, m}^{J}(c)$ is the $c$ th output value.

In the $\mathrm{CNN}$, the weights can be updated using the classic back-propagation algorithm, where SGD can be used for calculating the weight updates. In order to improve the training efficiency by allowing parallel computing, the local image-sets are split into multiple small subsets (or mini-batches) with the same mini-batch size denoted as $K$. Moreover, we assume that $\tau$ local updates are performed at each UAV. Explicitly, at the $i$ th local update, the $j$ th weight matrix $\boldsymbol{W}_{u, m}^{j,[i]}$ can be calculated as $\boldsymbol{W}_{u, m}^{j,[i]}=\boldsymbol{W}_{u, m}^{j,[i-1]}-\alpha \nabla \mathcal{L}\left(\boldsymbol{W}_{u, m}^{j,[i-1]}\right), i=1, \ldots, \tau$, where $\alpha$ is the learning rate and $\nabla \mathcal{L}\left(\boldsymbol{W}_{u, m}^{j,[i-1]}\right)$ denotes the corresponding gradient. Let us denote the resultant weight matrix as $\boldsymbol{W}_{u, m}^{j, t}=\boldsymbol{W}_{u, m}^{j,[\tau]}$, where $t=1, \ldots, T$ denotes the iteration index of the specific round of communication between each UAV and the GFC. Next, the resultant weight matrices of all the $U$ UAVs are sent to the GFC, as discussed below.

b) Global Aggregation: As shown in Section II-B, the local model updates of all the UAVs are sent to the GFC via the fading uplink. In FL, a weighted average of all the model weights has to be calculated at the GFC in order to obtain an updated global model. However, as inferred from (3), a multi-user detection scheme is required at the GFC to obtain the weights of each model, imposing a high computational complexity on the GFC. From another point of view, we observe from (3) that when the channel fades can be mitigated by TPC (i.e., $h_{u}=1, \forall u$ ), the received symbols shown in (3) become the desired weighted average impaired by AWGN in FL. In other words, with the aid of TPC, the received symbols in GFC actually represent the updated global model in FL. As a result, the GFC can simply broadcast its received symbols to all the UAVs without any further processing. Hence, in order to facilitate the global aggregation of all the $U$ models at the parameter server, a WZF TPC scheme is used by each UAV. Furthermore, time division duplexing (TDD) is adopted, where it may be assumed that the uplink and downlink CSI are identical owing to the channel's reciprocity. More explicitly, the CSI to be used by the TPC at each UAV is assumed to be known. Since the number of UAVs considered in our scenarios is small and single-antenna arrangements are assumed, orthogonal training signals can be used for channel estimation at the GFC, relying on the proposition of [25]. In this case, the CSI estimated at the $u$ th UAV can be modeled as $h_{u}=\hat{h}_{u}+e_{u}$, where $e_{u}$ denotes the channel estimation error of the GFC, which is then fed back to the $u$ th UAV. For simplicity, the CSI-error $e_{u}$ is assumed to obey a complex Gaussian distribution with zero mean and a unity variance of $\sigma_{e}^{2}$, where we have $0 \leq \sigma_{e}^{2}<1$. Hence, WZF based symbols transmitted by the $u$ th UAV can be expressed as

$$
\boldsymbol{X}_{u, m}^{j, t} \triangleq \lambda_{u}\left(\hat{h}_{u}^{*} /\left|\hat{h}_{u}\right|^{2}\right) \boldsymbol{W}_{u, m}^{j, t},
$$

where we have $\sum_{u=1}^{U} \lambda_{u}=1$. Upon substituting (6) into (3), we arrive at

$$
\begin{aligned}
\boldsymbol{R}_{m}^{j, t} & =\sum_{u=1}^{U} \lambda_{u} \frac{h_{u} \hat{h}_{u}^{*}}{\left|\hat{h}_{u}\right|^{2}} \boldsymbol{W}_{u, m}^{j, t}+\boldsymbol{N}_{m}^{j, t} \\
& =\sum_{u=1}^{U} \lambda_{u} \frac{\left(\hat{h}_{u}+e_{u}\right) \hat{h}_{u}^{*}}{\left|\hat{h}_{u}\right|^{2}} \boldsymbol{W}_{u, m}^{j, t}+\boldsymbol{N}_{m}^{j, t} \\
& =\sum_{u=1}^{U} \lambda_{u}\left(1+\frac{e_{u} \hat{h}_{u}^{*}}{\left|\hat{h}_{u}\right|^{2}}\right) \boldsymbol{W}_{u, m}^{j, t}+\boldsymbol{N}_{m}^{j, t} \\
& =\sum_{u=1}^{U} \lambda_{u} \boldsymbol{W}_{u, m}^{j, t}+\boldsymbol{E}_{m}^{j, t}+\boldsymbol{N}_{m}^{j, t},
\end{aligned}
$$

where we have $\boldsymbol{E}_{m}^{j, t} \triangleq \sum_{u=1}^{U} \lambda_{u} e_{u} \hat{h}_{u}^{*} \boldsymbol{W}_{u, m}^{j, t} /\left|\hat{h}_{u}\right|^{2}$. Here, it can be readily shown that the symbols of (7) received at the GFC are actually the weighted aggregation required by FL, implying that the GFC does not require any further signal processing procedue for the global aggregation.

Next, the global model update $\boldsymbol{R}_{m}^{j, t}$ is transmitted from the GFC to all the UAVs, where the weight matrix of the $u$ th UAV is updated as $\boldsymbol{W}_{u, m}^{j, t+1}=\boldsymbol{R}_{m}^{j, t}-\alpha \nabla \mathcal{L}\left(\boldsymbol{R}_{m}^{j, t}\right)$, which is used for the local update procedure at the $(t+1)$ st iteration. Finally, after $T$ iterations, the algorithm terminates. The proposed procedure is summarized in Algorithm 1.

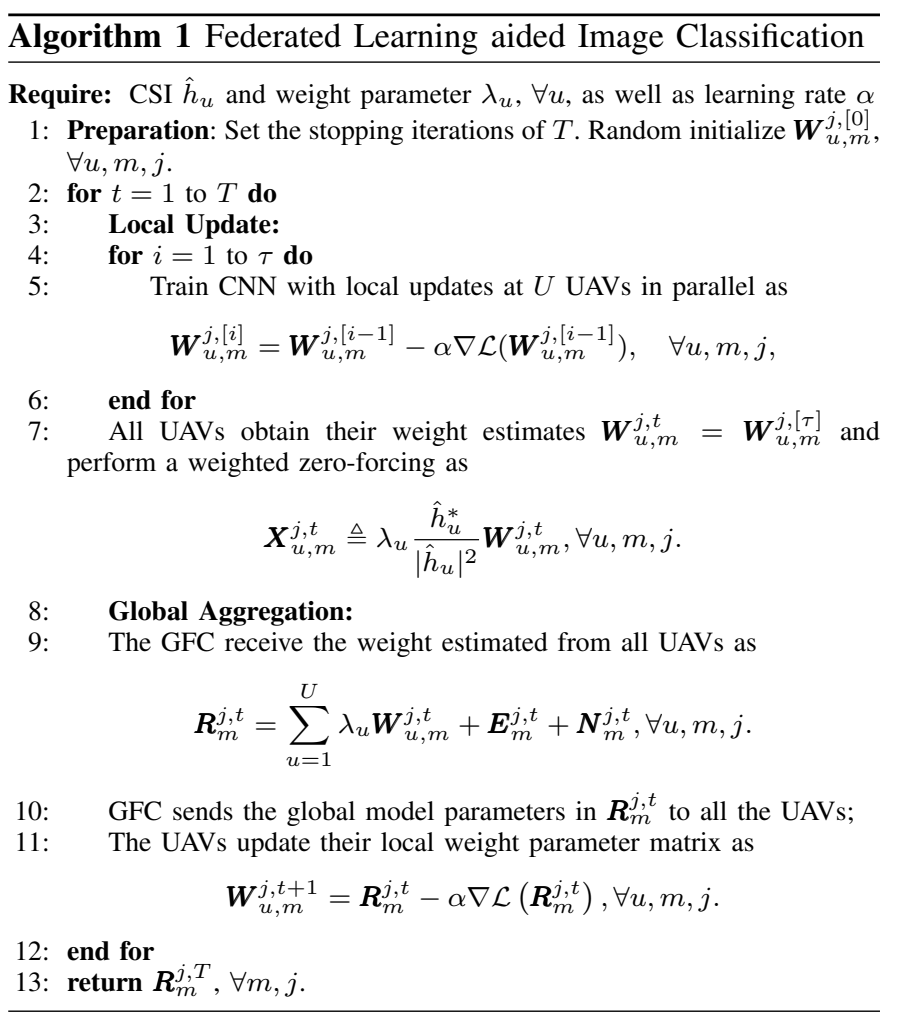

\section{Simulation Results}

In this section, simulation results are provided for characterizing the performance of the proposed FL-aided image classification task in multi-UAV networks. In our simulations, a 
total of $U=4$ UAVs hovering at $100 \mathrm{~m}$ height are considered. The distance between the $4 \mathrm{UAVs}$ and the GFC are $300 \mathrm{~m}$, $400 \mathrm{~m}, 800 \mathrm{~m}$, and 900 , respectively. The channel parameters of Section II-A are $\rho_{1}=4.88, \rho_{2}=0.43, \mu_{\mathrm{LoS}}=0.1$, $\mu_{\mathrm{NLOS}}=21$, and $\eta=2.2$. The transmitted power and the carrier frequency of each UAV are $P_{\mathrm{Tx}}=26 \mathrm{dBm}$ and $f_{\mathrm{c}}=2.4$ $\mathrm{GHz}$, respectively, while the noise power is $\sigma_{\mathrm{n}}^{2}=-97 \mathrm{dBm}$. By considering the limited computing capability of each UAV, a low-complexity CNN having a single convolutional layer, a pooling layer and a fully connected layer is trained at each UAV to carry out the image classification tasks based on $6 \times 10^{4}$ elements of the modified national institute of standard's (MNIST) handwritten sets [26]. Explicitly, $6 \times 10^{4}$ objects are randomly partitioned into 4 subsets, each containing $1.5 \times 10^{4}$ local training objects for each UAV. Furthermore, a total of $10^{4}$ objects are used as the test sets for characterizing the classification accuracy at the GFC. During each training, a total of 5420 weights are calculated at each UAV, i.e. we have $\left|\mathcal{W}_{1}\right|=\left|\mathcal{W}_{2}\right|=\left|\mathcal{W}_{3}\right|=\left|\mathcal{W}_{4}\right|=5420$, consisting of $(9 \times 9 \times 5+10 \times 500)=5405$ weights and $(5+10)=15$ bias values. In our simulations, the learning rate $\alpha$ is chosen from $[0.1,0.2, \ldots, 0.9]$ to obtain the best accuracy. Finally, an equal-weight parameter set of $\lambda_{1}=\lambda_{2}=\lambda_{3}=\lambda_{4}=1$ is assumed for the sake of simplicity in our simulations.

In Fig. 3 and Fig. 4, the classification accuracy of our FL-aided multi-UAV network aided image classification is investigated. In these figures, an error-free channel is assumed for each communication round. In Fig. 3, $\tau=1,2,4$ local updates are performed for each communication round with a mini-batch size of $K=1$ for SGD. Furthermore, each UAV performs $\tau=4$ local updates during each communication round with a mini-batch size of $K=1,10,20$ for SGD. Based on the simulation results of Fig. 3 and Fig. 4, we have the following observations. Firstly, as the number of local model updates increases, the classification accuracy is improved, as shown in Fig. 3. As a result, the total number of communication rounds required to achieve a target classification accuracy is reduced. However, the accuracy is saturating around 0.95. Secondly, as seen in Fig. 4, the classification accuracy of SGD relying on a higher mini-batch size converges faster, hence a smaller number of communication rounds is required for achieving a specific target classification accuracy upon increasing the mini-batch size of SGD used for local updates by the UAVs.

The accuracy of the FL-aided image classification in our multi-UAV network relying on imperfect CSI is investigated in Fig. 5. In this figure, the imperfect CSI model of Section III-B$\mathrm{b}$ is used, where the variance of channel errors is $\sigma_{e}^{2}=10^{-1}$, $10^{-2}, 10^{-2.5}, 10^{-3}, 10^{-4}$. Observe from Fig. 5 that the classification accuracy is reduced, when the CSI error is increased. As a result, a higher number of communication rounds is required for the proposed algorithm to attain a certain target accuracy, yielding a higher communication cost for the multi-UAV network considered. Furthermore, the classification accuracy can be hardly improved upon performing more communication rounds between the UAVs and the GFC for the case of $\sigma_{e}^{2}=10^{-1}$. This is not unexpected, since the procedure in Section III-B fails to accurately learn the image

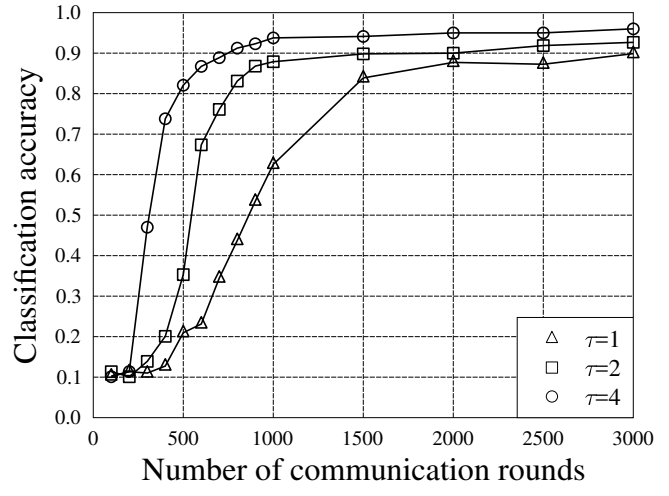

Fig. 3: Classification accuracy versus the number of communication rounds for our FL-aided image classification in multiUAV networks. Each UAV performs $\tau=1,2,4$ local updates for each communication round. The mini-batch size of the SGD is $K=1$.

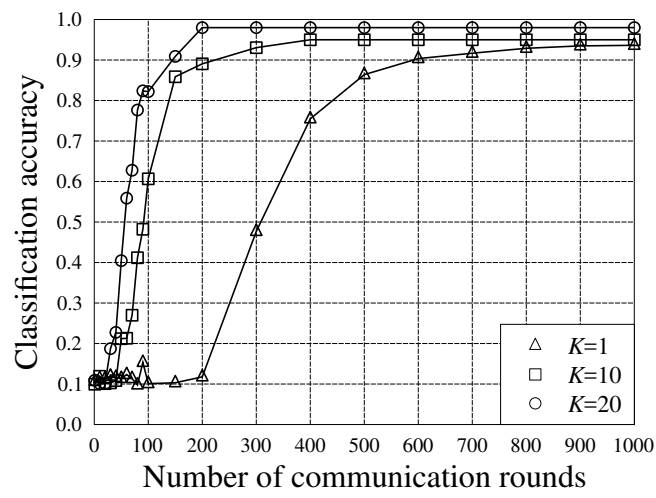

Fig. 4: Classification accuracy versus the number of communication rounds for our FL-aided image classification in multiUAV networks. Each UAV performs $\tau=4$ local updates for each communication round. The mini-batch size of the SGD is $K=1,10,20$.

classification model, when the global updates shown in (7) are impaired by CSI errors.

TABLE I: Transmission time comparison of centralized learning and FL-aided image classification

\begin{tabular}{l|c|c|c|c}
\hline \hline classification accuracy & 0.76 & 0.82 & 0.9 & 0.95 \\
\hline Centralized Learning & $3.1 \mathrm{~s}$ & $4.7 \mathrm{~s}$ & $6.3 \mathrm{~s}$ & $9.4 \mathrm{~s}$ \\
\hline FL-IC & $35 \mathrm{~ms}$ & $39 \mathrm{~ms}$ & $52 \mathrm{~ms}$ & $65 \mathrm{~ms}$ \\
\hline \hline
\end{tabular}

Finally, the transmission time of image classification relying on centralized learning and that of FL-aided image classification (i.e., FL-IC shown in Table I) are compared in Table I. Specifically, for the centralized learning shown in Table I, the locally collected datasets are directly transmitted to the GFC by $4 \mathrm{UAVs}$, where the same $\mathrm{CNN}$ is employed at the GFC for performing image classification. Furthermore, $\tau=50$ local updates are performed for each communication round with a mini-batch size of $K=20$ for SGD, and imperfect CSI associated with a variance of $10^{-3}$ is used. 


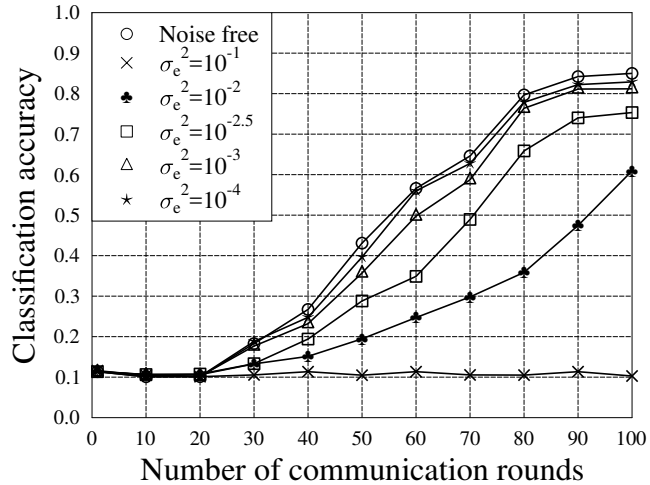

Fig. 5: Classification accuracy versus the number of communication rounds for FL-aided image classification in multi-UAV networks. Each UAV performs $\tau=4$ local updates for each communication round. The mini-batch size of SGD is $K=20$.

An uplink transmission rate of $10 \mathrm{Mbps}$ is assumed. As seen in Table I, for a given target classification accuracy, the time required for uplink transmission can be significantly reduced by the proposed FL-aided image classification in comparison to that of the Non-FL case, albeit this is achieved at a higher computing cost at each UAV.

\section{CONCLUSIONS}

This paper studied image classification in area exploration scenarios, where a multi-UAV system has been conceived. Specifically, a GFC having limited computing-cachingcommunication capability has been employed for the coordination of UAVs in our system. In the proposed system, FL has been invoked for accomplishing the image classification tasks, based on the local model learned from images collected by an on-board camera at each UAV. In the proposed FL-aided classification, the local updates of all UAVs are transmitted to the GFC via fading wireless channels. Then, a global update is performed at the GFC and the corresponding results are fed back to UAVs for performing the next round of local model update. In order to facilitate global update at the GFC, a WZF TPC scheme has been proposed, which relied on imperfect CSI. Our results have shown that for a given target test accuracy, the proposed FL-aided system is capable of operating at a much lower communication cost, than its centralized learning aided counterpart.

\section{REFERENCES}

[1] J. Zhang, T. Chen, S. Zhong, J. Wang, W. Zhang, X. Zuo, R. G. Maunder, and L. Hanzo, "Aeronautical ad hoc networking for the internet-abovethe-clouds," Proceedings of the IEEE, vol. 107, pp. 868-911, May 2019.

[2] Y. Yang, Z. Zheng, K. Bian, L. Song, and Z. Han, "Real-time profiling of fine-grained air quality index distribution using UAV sensing," IEEE Internet of Things Journal, vol. 5, no. 1, pp. 186-198, 2018.

[3] F. Tang, Y. Kawamoto, N. Kato, and J. Liu, "Future intelligent and secure vehicular network toward 6G: Machine-learning approaches," Proceedings of the IEEE, vol. 108, no. 2, pp. 292-307, 2020.

[4] K. P. Valavanis and G. J. Vachtsevanos, Handbook of unmanned aerial vehicles. Springer Netherlands, 2015
[5] Y. Zeng, R. Zhang, and T. J. Lim, "Wireless communications with unmanned aerial vehicles: opportunities and challenges," IEEE Communications Magazine, vol. 54, no. 5, pp. 36-42, May 2016.

[6] F. Tang, Z. M. Fadlullah, N. Kato, F. Ono, and R. Miura, "AC-POCA: Anticoordination game based partially overlapping channels assignment in combined UAV and D2D-based networks," IEEE Transactions on Vehicular Technology, vol. 67, no. 2, pp. 1672-1683, 2018.

[7] Y. Yang, Z. Zheng, K. Bian, Y. Jiang, L. Song, and Z. Han, "Arms: A fine-grained 3D AQI realtime monitoring system by UAV," in GLOBECOM 2017 - 2017 IEEE Global Communications Conference, 2017, pp. $1-6$.

[8] M. Nguyen, L. D. Nguyen, T. Q. Duong, and H. D. Tuan, "Realtime optimal resource allocation for embedded UAV communication systems," IEEE Wireless Communications Letters, no. 1, Feb 2019.

[9] T. Bai, J. Wang, Y. Ren, and L. Hanzo, "Energy-efficient computation offloading for secure UAV-edge-computing systems," IEEE Transactions on Vehicular Technology, vol. 68, no. 6, pp. 6074-6087, June 2019.

[10] X. Liu, Y. Liu, Y. Chen, and L. Hanzo, "Trajectory design and power control for multi-uav assisted wireless networks: A machine learning approach," IEEE Transactions on Vehicular Technology, vol. 68, no. 8, pp. 7957-7969, Aug 2019.

[11] T. Q. Duong, L. D. Nyuyen, H. D. Tuan and L. Hanzo, "Learningaided realtime performance optimisation of cognitive UAV-assisted disaster communication," in IEEE Global Communications Conference(GLOBECOM'19), Dec. 2019.

[12] J. Konecny, H. B. Mcmahan, D. Ramage, and P. Richtarik, "Federated optimization: Distributed machine learning for on-device intelligence," arXiv preprint arXiv:1610.02527, 2016.

[13] J. Konecny, H. B. Mcmahan, F. X. Yu, P. Richtarik, A. T. Suresh, and D. Bacon, "Federated learning: Strategies for improving communication efficiency," arXiv preprint arXiv:1610.05492, 2016.

[14] S. Wang, T. Tuor, T. Salonidis, K. K. Leung, C. Makaya, T. He, and K. Chan, "Adaptive federated learning in resource constrained edge computing systems," IEEE Journal on Selected Areas in Communications, vol. 37, no. 6, pp. 1205-1221, June 2019.

[15] J. Ren, H. Wang, T. Hou, S. Zheng, and C. Tang, "Federated learningbased computation offloading optimization in edge computing-supported Internet of Things," IEEE Access, vol. 7, pp. 69 194-69201, 2019.

[16] J. Kang, Z. Xiong, D. Niyato, S. Xie, and J. Zhang, "Incentive mechanism for reliable federated learning: A joint optimization approach to combining reputation and contract theory," IEEE Internet of Things Journal, vol. 6, no. 6, pp. 10700-10714, Dec 2019

[17] H. H. Yang, Z. Liu, T. Q. S. Quek, and H. V. Poor, "Scheduling policies for federated learning in wireless networks," IEEE Transactions on Communications, pp. 1-1, 2019.

[18] S. Samarakoon, M. Bennis, W. Saad, and M. Debbah, "Distributed federated learning for ultra-reliable low-latency vehicular communications," IEEE Transactions on Communications, pp. 1-1, 2019.

[19] S. Zhang, H. Zhang, Q. He, K. Bian, and L. Song, "Joint trajectory and power optimization for UAV relay networks," IEEE Communications Letters, vol. 22, no. 1, pp. 161-164, 2018.

[20] A. Al-Hourani, S. Kandeepan, and S. Lardner, "Optimal LAP altitude for maximum coverage," IEEE Wireless Communications Letters, vol. 3 , no. 6, pp. 569-572, Dec 2014.

[21] H. Jiang, Z. Zhang, and G. Gui, "Three-dimensional non-stationary wideband geometry-based UAV channel model for A2G communication environments," IEEE Access, vol. 7, pp. 26116-26 122, 2019.

[22] D. W. Matolak and R. Sun, "Air-ground channel characterization for unmanned aircraft systems Part I: Methods, measurements, and models for over-water settings," IEEE Transactions on Vehicular Technology, vol. 66, no. 1, pp. 26-44, Jan 2017.

[23] R. Rajashekar, M. Di Renzo, K. V. S. Hari, and L. Hanzo, "A beamforming-aided full-diversity scheme for low-altitude air-to-ground communication systems operating with limited feedback," IEEE Transactions on Communications, vol. 66, no. 12, pp. 6602-6613, Dec 2018.

[24] L. Hanzo, M. Münster, B. Choi, and T. Keller, $O F D M$ and MC-CDMA for Broadband Multi-user Communications, WLANs and Broadcasting. John Wiley \& Sons, May 2003.

[25] B. Hassibi and B. M. Hochwald, "How much training is needed in multiple-antenna wireless links?" IEEE Transactions on Information Theory, vol. 49, no. 4, pp. 951-963, 2003.

[26] C. C. Y. LeCun and C. Burges, "The MNIST database of handwritten digits," http://yann.lecun.com/exdb/mnist, 1998. 